\title{
Development of a High-Speed Digitizer to Time Resolve Nanosecond Fluorescence Pulses
}

\author{
E. Moreno-García* ${ }^{* 1}$ R. Galicia-Mejía ${ }^{2}$, D. Jiménez-Olarte ${ }^{3}$, J. M. de la Rosa-Vázquez ${ }^{4}$, S. Stolik-Isakina ${ }^{5}$ \\ ${ }^{1,3}$ Laboratorio de Instrumentación Electrónica, ESFM-IPN \\ Edificio No.9, U.P. "Adolfo López Mateos", 07630 México D.F. \\ Tel. 57296000 ext. 55007 \\ *emoreno@esfm.ipn.mx \\ 2,4,5 Laboratorio de Láseres. SEPI-ESIME, IPN \\ Edificio No.5, U.P. "Adolfo López Mateos", 07630 México D.F. \\ Tel. 57296000 ext. 54622
}

\begin{abstract}
The development of a high-speed digitizer system to measure time-domain voltage pulses in nanoseconds range is presented in this work. The digitizer design includes a high performance digital signal processor, a high-bandwidth analog-to-digital converter of flash-type, a set of delay lines, and a computer to achieve the time-domain measurements. A program running on the processor applies a time-equivalent sampling technique to acquire the input pulse. The processor communicates with the computer via a serial port RS-232 to receive commands and to transmit data. A control program written in LabVIEW 7.1 starts an acquisition routine in the processor. The program reads data from processor point by point in each occurrence of the signal, and plots each point to recover the time-resolved input pulse after $n$ occurrences. The developed prototype is applied to measure fluorescence pulses from a homemade spectrometer. For this application, the LabVIEW program was improved to control the spectrometer, and to register and plot time-resolved fluorescence pulses produced by a substance. The developed digitizer has $750 \mathrm{MHz}$ of analog input bandwidth, and it is able to resolve 2 ns rise-time pulses with 150 ps of resolution and a temporal error of 2.6 percent.
\end{abstract}

Keywords: nanosecond pulses, digitizer, time-equivalent sampling, time-resolved fluorescence.

\section{RESUMEN}

En este trabajo se presenta el desarrollo de un sistema digitalizador de alta velocidad para medir en el dominio del tiempo pulsos de voltaje del orden de nanosegundos. El diseño del digitalizador incluye un procesador digital de señales de alto rendimiento, un convertidor analógico digital tipo flash con ancho de banda grande, un conjunto de líneas de retardo, y una computadora para realizar las mediciones en el dominio del tiempo. El procesador ejecuta un programa que aplica una técnica de muestreo en tiempo equivalente para adquirir el pulso de entrada. El procesador se comunica con la computadora vía un puerto serie RS-232 para recibir comandos y transmitir datos. Se desarrolló un programa de control en LabVIEW 7 para iniciar una rutina de adquisición en el procesador. El programa lee datos del procesador punto por punto en cada ocurrencia de la señal, y grafica cada punto para recuperar el pulso de entrada resuelto en tiempo después de $n$ ocurrencias. El prototipo desarrollado se aplica para medir pulsos de fluorescencia provenientes de un espectrómetro. Para esta aplicación se amplió el programa en LabVIEW para controlar el espectrómetro, y para registrar y graficar pulsos de florescencia resuelta en tiempo producidos por una substancia. El digitalizador desarrollado tiene un ancho de banda de $750 \mathrm{MHz}$ en su entrada analógica, y es capaz de resolver pulsos con tiempo de subida de 2 ns con una resolución de 150 ps y un error temporal de 2.6 percent.

\section{Introduction}

With the advancement of high transmission-rate systems and short laser pulses, many applications now require high time-resolution measurement instruments. The applications of picoseconds timedomain measurements are being increased because of the expansion of digital methods and pulse techniques in many scientific areas. In chemical, physical and biological experiments, picosecond and femtosecond ultra-fast laser systems are becoming increasingly common as diagnosis tools for studying fundamentals physics processes. An automatic process for high timeresolution measurements involves the use of a programmable digital system or digitizer. This could be a stand-alone instrument, or a digital oscilloscope, or a computer combined with a digital 
signal processor (DSP), for example. Particularly, we are interested in time-resolved fluorescence spectroscopy, where optical pulses of nanosecond order have to be recorded. For this application we designed a low-cost high-speed digitizer.

The fluorescence phenomenon occurs when a specimen is excited with light, which is absorbed by the specimen and then reemitted. The emitted fluorescence contains information about the specimen under study. For time-resolved fluorescence, the excitation is a very short light pulse (ideally an impulse function $\delta(t)$ ), and the fluorescence response pulses have widths and rise times of nanosecond order. These optical pulses are converted to electrical pulses by means of photodetectors. The spectrofluorometry is related to the measuring of the emission spectra produced by fluorescent specimens, it is the most extensively used optical spectroscopic method in analytical measurements and scientific investigation in the measurement of species concentrations in gases, liquids and solids [1, 5]. The fluorescence spectroscopy is being widely used nowadays for biomedical purposes and clinical analysis, among other many applications. In vivo spectrofluorometry has emerged as a powerful technique for biomedical research covering a broad spectrum, from the study of cellular and tissue structures, to the study of the biological function and early detection of cancer. Biomedical fluorescence spectroscopy is an extremely large and growing field of research $[6,9]$.
Fluorescence measurements can be broadly classified into two types: steady-state and timeresolved. Steady-state measurements are those performed with constant illumination and observation. This is the most common type of measurement. The sample is illuminated with a continuous beam of light and the intensity of the emission is usually recorded as wavelength function (fluorescence spectrum). Time-resolved measurements are used for measuring intensity decays. For those measurements, the sample is exposed to a pulse of light, where the pulse width is typically shorter than the decay time of the sample. The intensity decay is recorded with a high speed detection system that permits the intensity to be measured on the nanosecond time scale. Timedependent measurements resolve fluorescence intensity decay in terms of lifetimes, and thus provide additional information about the intra and intermolecular dynamics of the fluorophore (the fluorescent compound of a substance) under study $[1,2,10]$.

Figure 1 shows a general structure of a spectrofluorometer to measure steady-state, as well as time-resolved fluorescence. Usually, the sample under study is excited with ultraviolet light pulses, the sample emits fluorescence in all directions, and part of this emission is captured at the monochromator input through $90^{\circ}$ geometry. The monochromator output is a light with a single wavelength $(\lambda)$ component of the input light, coming from the sample. The light pulse from the

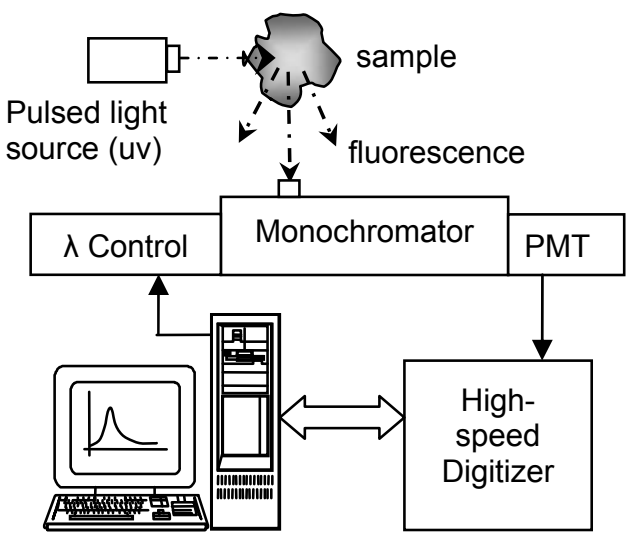

Figure 1. General structure of a spectrofluorometer. 
monochromator output is detected by a photomultiplier tube (PMT) and converted to a current pulse, which is acquired by a high-speed digitizer. A program running on the computer controls the operation of both, the monochromator and the digitizer. The computer sends commands to the digitizer and also reads and plots the measured signals.

In this work we report the design and development of a high-speed digitizer to be applied as acquisition system in time-resolved fluorescence spectroscopy. For this application we use a homemade spectrometer [11] with a monochromator of the Czerny-Turner type with focal length of $275 \mathrm{~mm}$, and a PMT module H7732P-11 from Hamamatsu Inc. (80 mA/W peak radiant sensitivity at $430 \mathrm{~nm}$ and 2 ns characteristic rise time). The monochromator output wavelength is controlled via a USB port by means of the USB-6009 DAQ board from National Instruments. The spectrometer operation is automated with a program developed in LabVIEW 7.1 to perform wavelength sweeps from $200 \mathrm{~nm}$ to $800 \mathrm{~nm}$. As pulsed light source we use the drive module PC0-7810 from Direct Energy Inc. which produces a differential current pulse, up to 40 A with 4 ns full width half maximum (FWHM). This current pulse is applied to the UV LED NSHU033A manufactured from Nichia Co., with emission peak at $365 \mathrm{~nm}$, and a continuous radiation power of $210 \mathrm{~mW}$. The drive module can be externally triggered to generate light pulses from UV LED.

\section{Sampling Techniques}

An automatic process for time-domain measurements involves the use of a programmable digital system, thus the continuous time signal pulse to measure has to be converted to a digital format in order to be recognized by the digital system. Although there are a number of different implementations of sampling technology, there are two basic methods: real-time sampling and equivalent-time sampling. Equivalent-time sampling can be divided further, into two sub-categories: random and sequential. Each method has distinct advantages depending on the kind of measurements being made. Real-time methods require only a single occurrence of the signal to acquire an entire waveform, and the digitizer or sampler operates at maximum speed to acquire as many points as possible in one sweep. Equivalenttime methods, on the other hand, require multiple occurrences of the signal to acquire the waveform. Samples are acquired over many repetitions of the signal, with one or more samples taken on each repetition $[12,13]$.

The sequential equivalent-time technique acquires one sample per period signal, and includes a trigger signal as Figure 2 shows. When the first trigger is detected, the first sample is taken after a fixed initial time T1. At the next period, a second sample is taken after a very short, but well-defined delay $\Delta t$ from the time position of the first sample. When the next trigger occurs, the same time $\Delta t$ is added to the previous delay and another sample is

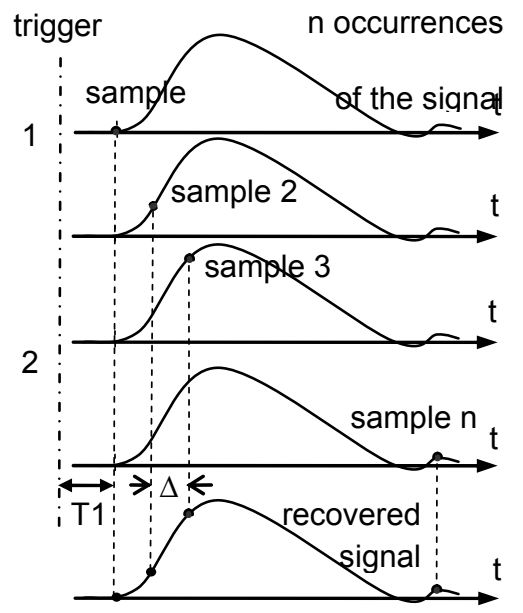

Figure 2. Sequential equivalent-time sampling. 
taken. This process is repeated many times, and after $\mathrm{n}$ periods of the signal, $\mathrm{n}$ samples have been taken to recover the waveform of the signal. If the sample point is shifted in sufficiently small steps $\Delta t$, this sampling method provides very high bandwidths and timing resolution needed for timeresolved fluorescence signals, with an extremely high virtual sample rate $=1 / \Delta$ t. To improve the signal-to-noise ratio the equivalent-time sampling method can be combined with multiple time averaging method. For that purpose, the procedure described above is repeated several times and the obtained waveforms are averaged by the digital system. Averaging of $n$ curves reduces the noise error by a factor of $\mathrm{n}^{-1 / 2}$. In the "boxcar" method at each sample point $n$ samples are taken and averaged with the same delay setting. When the averaging for this signal point is complete the delay is increased by $\Delta \mathrm{t}$. The resulting noise error is also reduced by $\mathrm{n}^{-1 / 2}$ [14].

\section{Digitizer Design}

Particularly we are interested in time resolving fluorescence lifetimes on the nanosecond time scale. For these very short times there are two important specifications of the acquisition system behavior to consider: its analog front-end bandwidth (BW) and its equivalent rise time (tr). Bandwidth describes the analog front end's ability to capture an analog signal with minimal amplitude loss, and rise time slowing. Bandwidth is defined as the frequency at which the amplitude of the sampled signal decays 30 percent due to ADC performance. The rise time for a signal is defined as the time to transit from 10 percent to 90 percent of the maximum signal amplitude. Both concepts can be applied in general to signals, to systems, or to components, and they are inversely related by Equation (1), which is based on the one pole model, R-C limited input response.

$$
\mathrm{BW}(\operatorname{dig})=\frac{0.35}{\operatorname{tr}(\operatorname{dig})}
$$

The bandwidth BW(dig) or rise time tr(dig) of the digitizer analog front end, introduces an amplitude loss and a rise time slowing of the signal to measure. In practice, it is recommended that the bandwidth of the digitizer be 3 to 5 times the signal bandwidth $\mathrm{BW}(\mathrm{sig})$ to reduce the amplitude error near 3 percent to 1 percent. The measured rise time can be estimated by using a sum-of-squares technique as Equation (2), where $\operatorname{tr}(\mathrm{sig})$ is the rise time of the signal.

$$
\operatorname{trm}^{2}=\operatorname{tr}^{2}(\mathrm{dig})+\operatorname{tr}^{2}(\mathrm{sig}
$$

In time-resolved fluorescence spectroscopy, the monochromator and photodetector of Figure 1 also contribute to slowing the measured signal. Thus Equation (2) must include the characteristic rise-time terms of each optical device.

Figure 3 shows a schematic diagram of the digitizer developed with three main blocks. We choose a high performance digital signal processor (DSP) from Microchip Technology Inc. (dsPIC33FJ256GP710), as a programmable digital system to perform the equivalent-time sampling technique. The processor operates at $40 \mathrm{MHz}$ clock frequency and contains $250 \mathrm{~KB}$ of program flash memory and $30 \mathrm{~KB}$ of data SRAM. This DSP provides a variety of resources; in our application we use 5 output control lines, 12 input lines for data, one timer, and a serial interface.

A program running on the DSP generates a SYNC pulse to synchronize the experiment with the instrument. In our experiment, the SYNC pulse triggers the UV light source, and the fluorescence response pulse from the PMT is acquired by the analog-to-digital converter module. For this module we use an evaluation board of the integrated circuit MAX12528 from Maxim/Dallas, which contains two high-speed 12-bit ADCs with $750 \mathrm{MHz}$ full-power analog input bandwidth. This board includes a passive input stage with an inductive coupling by means of a pulse transformer for both ADCs. This input circuit converts the current pulse generated by the PMT to a differential voltage pulse required at the analog input of the integrated circuit MAX12528. Both converters acquire values continuously on each rising edge of the sampling clock input SCK. The voltage value present at analog inputs $( \pm 1.024 \mathrm{~V}$ input range), during the rising edge of the sampling clock, is acquired and converted to a digital format. The digital output value is available on 12 data lines (DATA) after 8 rising edges of the sampling clock. In this application we use only one converter. 
To apply the sequential equivalent-time sampling technique we use three programmable 8-bit delay lines (DS1020-A15 from Maxim/Dallas). These circuits are used to program a delay $n \Delta t$ of the oscillator signal CK to produce a delayed clock signal CKDL. With the rising edge of this delayed signal, the voltage value present at input AIN is sampled. The delay values of each delay line can be varied over 255 equal $n$ steps of 0.15 ns each one, with a maximum delay of $38.25 \mathrm{~ns}$. The three delays lines connected in cascade allows programming up to a maximum delay of $114.75 \mathrm{~ns}$ $(n=765)$. The delay integer $n$ is programmed in series mode with five output lines from the DSP.

Figure 4 shows the temporal relation of the signals during the equivalent-time sampling process of three points. A program running on the DSP generates the oscillator signal $\mathrm{CK}(5 \mathrm{MHz})$ and a SYNC pulse with programmable repetition rate (we use $40 \mathrm{KHz}$ ). The SYNC pulse triggers the experiment, and the response pulse from the experiment always occurs at the same time respect to SYNC. The rising edge of the delayed clock signal CKDL, samples one value of the analog input after each occurrence of SYNC. For $n=0$ the first input signal value is acquired, the program waits for $8 \mathrm{CKDL}$ pulses, then reads the digitized value DATA of that sample and increments the delay integer $\mathrm{n}$ by one. Now the sampling clock is delayed $\mathrm{n} \Delta \mathrm{t}=0.15 \mathrm{~ns}$. The program sends the previous digitized value to the computer to be stored and graphed, and generates the next SYNC pulse. The second value is sampled during the next rising edge of CKDL after SYNC, this occurs 0.15 ns after the previous value of input signal, and the program repeats the procedure with the sampled value. When next SYNC occurs, $n=2$ and an input value is sampled 0.3 ns apart from SYNC.

The process is repeated many times until $n=765$ and $n \Delta t=114.75 \mathrm{~ns}$, which is the time range of the digitizer. The minimum sampling rate specified for the MAX12528 is $5 \mathrm{MHz}$, thus we use this frequency for the clock signal to have a period of 200 ns, which is longer than time range as required. The digitizer sampling rate is given by $\Delta t$ between samples and not by the ADC sampling rate, that is $1 / 0.15 \mathrm{~ns}=6.66 \mathrm{GS} / \mathrm{s}$. Each acquired value is sent to the computer before to acquire the next value. A control program was developed in the graphical language $\mathrm{G}$ of LabVIEW 7 to start an acquisition routine in the DSP. The program reads data from processor point by point, and plots the time-resolved input pulse. For noise reduction by averaging, the program can command the DSP to perform a number $\mathrm{k}$ of acquisitions with the same delay setting $\mathrm{n} \Delta \mathrm{t}$.

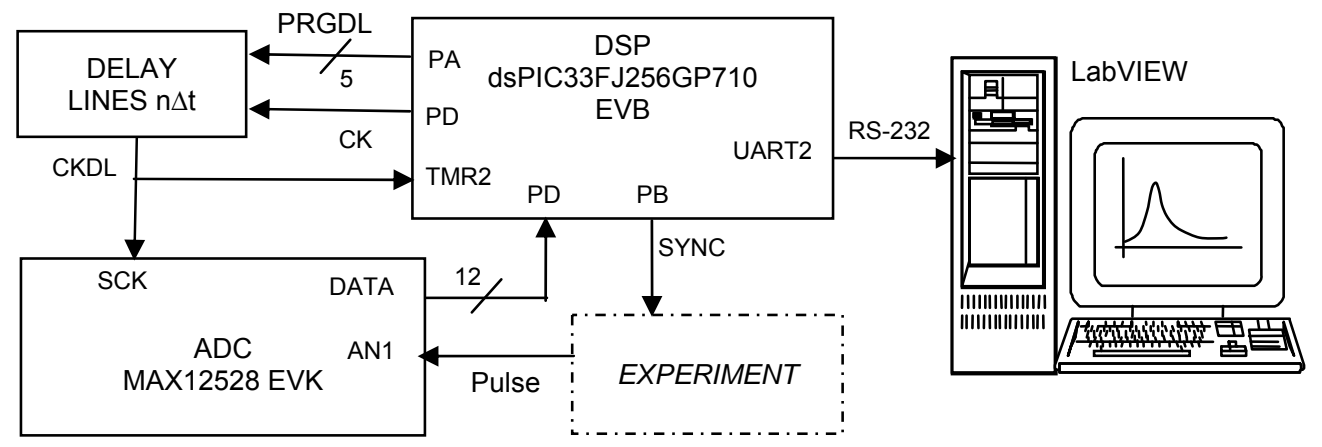

Figure 3. Schematic diagram of the digitizer developed. 


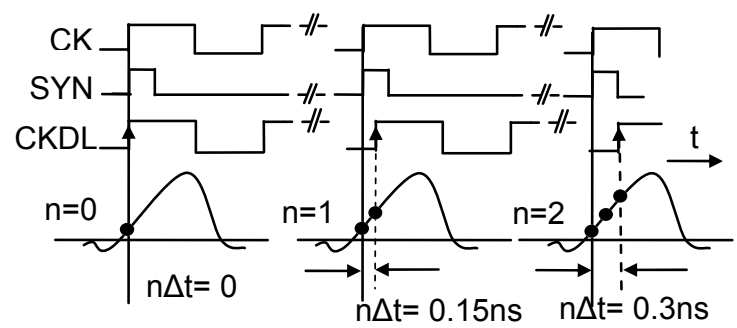

Figure 4. Acquisition of three points during equivalent-time sampling process.

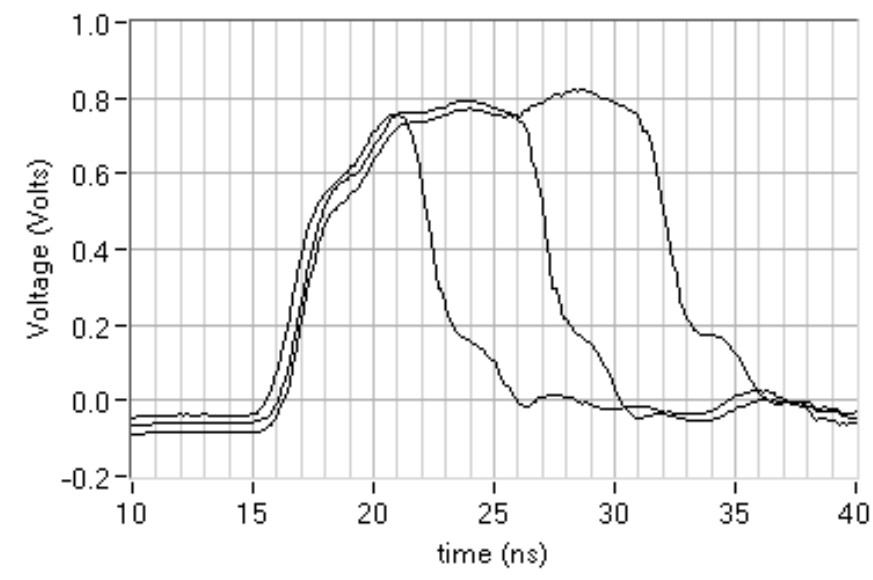

Figure 5. Three test pulses measured averaging 10 samples with the same delay $\mathrm{n} \Delta \mathrm{t}$ setting.

\section{Results and Discussion}

The digitizer developed was successfully tested during time-domain measurements of voltage pulses with known parameters. We use three test pulses generated by the high-speed programmable monostable DS1040Z-A15 from Maxim/Dallas, with FWHM of 5,10 and $15 \mathrm{~ns} \pm 5$ percent, and $4 \mathrm{~ns}$ rise time. The SYNC pulse in Figure 6 triggers the monostable circuit at a repetition rate of $40 \mathrm{KHz}$. Figure 5 shows the measured test pulses averaging 10 samples per point. From the data measured, the pulse widths observed are approximately $5.2 \mathrm{~ns}, 10.1 \mathrm{~ns}$ and $15.2 \mathrm{~ns}$. These times are greater than expected because the digitizer introduces a signal slowing, as well as the assembly connections at digitizer input. The maximum deviation is 4 percent $(0.2 \mathrm{~ns})$ for $5 \mathrm{~ns}$ test pulse considering the nominal value specified.
According to Equation (1) for $750 \mathrm{MHz}$ analog input bandwidth, the digitizer characteristic rise time is $\operatorname{tr}(\mathrm{dig})=0.466 \mathrm{~ns}$, and the input signal rise time is 4 ns. Thus from Equation (2), the expected rise time to measure is trm $=4.027 \mathrm{~ns}$. From data in Figure 7 the three test pulses have approximately $4.04 \mathrm{~ns}$ rise time, which is 0.32 percent greater than expected value, and again this is because the signal slowing introduced by input assembly connections.

Figure 6 shows the 10 ns test pulse measured with the digitizer development (continuous line), with a no-attenuated probe and an oscilloscope (dash line), and with an attenuated probe and oscilloscope (short-dash line). For the measurement with the digitizer (averaging 10 samples per point) a well-defined pulse is observed, meanwhile, for the measurements with the oscilloscope and probes, we observe 


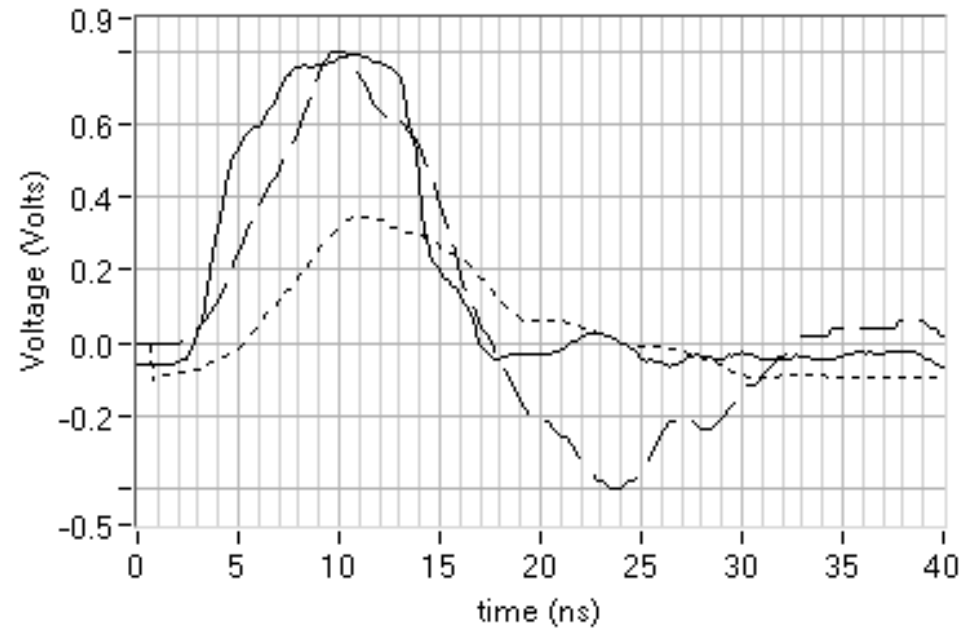

Figure 6. Test pulse of 10ns FWHM measured with the digitizer (continuous line), with no-attenuated probe and oscilloscope (dash line) and with attenuated probe and oscilloscope (short-dash line).

attenuation and distortion of the signal measured. With both probes (attenuated and no-attenuated) the measured pulses have a FWHM near of 9 ns and a slower rise time around $5.6 \mathrm{~ns}$. The attenuated probe causes almost 50 percent of amplitude attenuation of the signal and the pulse is not well-defined. This is because the probe used has a bandwidth of only $100 \mathrm{MHz}$, which is not enough to reproduce the high frequency components of the input pulse. The oscilloscope used is a Tektronix digital oscilloscope, model 2440 , with $300 \mathrm{MHz}$ of input bandwidth, or equivalent rise time $\operatorname{tr}(\mathrm{osc})=1.66 \mathrm{~ns}$, according to Equation (1). This produces a slower rise time measured by the oscilloscope as we can appreciate in the dash line trace of Figure 6.

A quantitative measure of the quality of a digitizer output signal is the signal-to-noise ratio (SNR), which is defined as the ratio of the information signal power to the unwanted noise power. The larger the number, the better it can differentiate the signal from noise. Both signal and noise power must be measured at the same points in a system, and within the same system bandwidth. If the signal and the noise are measured across the same impedance, then the SNR can be obtained by calculating the square of the amplitudes ratio as Equation (3).

$$
\mathrm{SNR}=\frac{\text { Psignal }}{\text { Pnoise }}=\left(\frac{\text { Asignal }}{\text { Anoise }}\right)^{2}
$$

Without triggering the test monostable circuit, the measured peak-to-peak electrical noise, averaging 10 samples per point, was about $12 \mathrm{mV}$, and the peak-to-peak amplitude of the $10 \mathrm{~ns}$ test pulse is about $0.86 \mathrm{~V}$. From Equation (3) we calculate SNR $=71.75$. This value is reduced when the digitizer is used to acquire data from a real experiment, which introduces more noise than the test circuit.

Figure 7 shows two signals measured with the developed instrument averaging 6 samples per point. The negative pulse (dash line) corresponds to a voltage pulse which is proportional to the current pulse (36 A peak value) applied to the UV LED used as light source. For this pulse we can observe a 2 ns rise time (fall time), and 5 ns FWHM. The other signal (continuous line) is the optical pulse emitted by the UV LED, which is detected by the PMT trough the spectrometer at $365 \mathrm{~nm}$. Figure 9 shows a second current pulse around $28 \mathrm{~ns}$, which produces a second peak of the optical pulse near $58 \mathrm{~ns}$. This unwanted oscillation in the current signal, which is applied to the LED, could be caused by parasite inductances, LED capacitance and connections resistance of the LED and the drive module PC0-7810. This defect of the pulsed UV light source limits the instrument capacity to measure fluorescence decays. The optical pulse in Figure 9 has a rise time around 8 ns, and assuming an UV light pulse at spectrometer input with 2 ns rise time (same of the current pulse), thus from Equation (2), the characteristic rise time of the complete instrument 
(monochromator + PMT + Digitizer) is of $7.75 \mathrm{~ns}$. This slowing of the input signal is meanly caused by the monochromator, since $\operatorname{tr}(\mathrm{PMT})=2 \mathrm{~ns}$ and $\operatorname{tr}(\operatorname{dig})=0.46 \mathrm{~ns}$.

For time-resolved fluorescence measurements, a program was developed in the graphical language G of LabVIEW 7.1 to control the instrument and to register and plot the time-resolved fluorescence pulses, as well as the fluorescence spectrum. Several substances were irradiated with UV light pulses at $365 \mathrm{~nm}$. The luminous responses of the substances were collected into monochromator input through a $90^{\circ}$ geometry. The control program performs a wavelength sweep in an interval defined by the user. The user also defines the step-sweep value $\Delta \lambda$. For each value of $\lambda$ the program initiates the digitizer routine in the DSP to perform a complete temporal sweep from 0 ns to $114.75 \mathrm{~ns}$ of the fluorescence pulse emitted by the substance. The peak value of this pulse represents for that $\lambda a$ single point of the steady-state fluorescence spectrum. Figure 8 shows the time-resolved measurements obtained from white color bond paper for three different $\lambda$ values. The fluorescence spectrum in Figure 9 was constructed with the maximum values of time-resolved measurements, for each wavelength. Figure 10 and Figure 11 show similar fluorescence measurements obtained from antifreeze. Time-resolved signals in Figures 8 and 10 show the effect of the light source unwanted oscillation around $50 \mathrm{~ns}$. These signals completely decay $40 \mathrm{~ns}$ after they start to increase and they show a similar behavior of the excitation optical pulse.

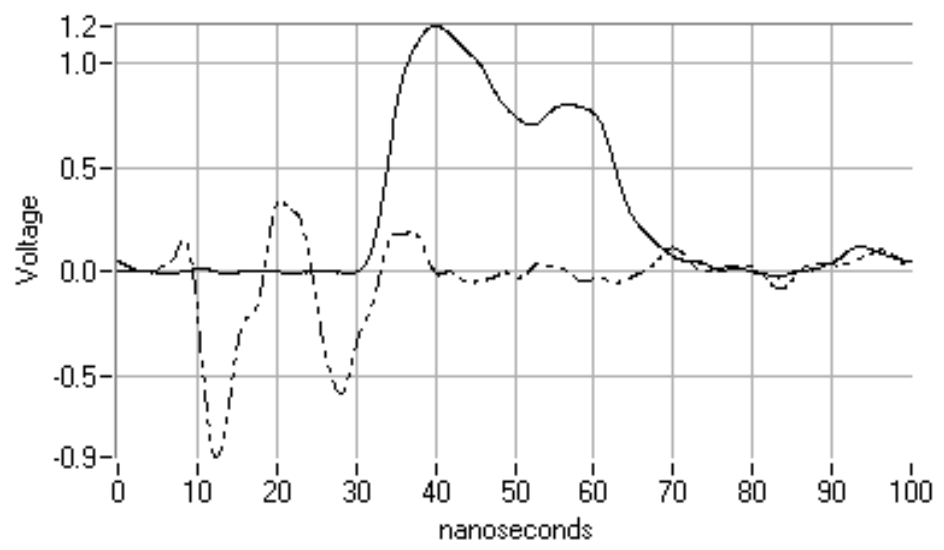

Figure 7. Current pulse applied to the UV LED (dash trace) and optical pulse emitted by the LED, and measured with the spectrometer and the digitizer prototype (continuous trace)

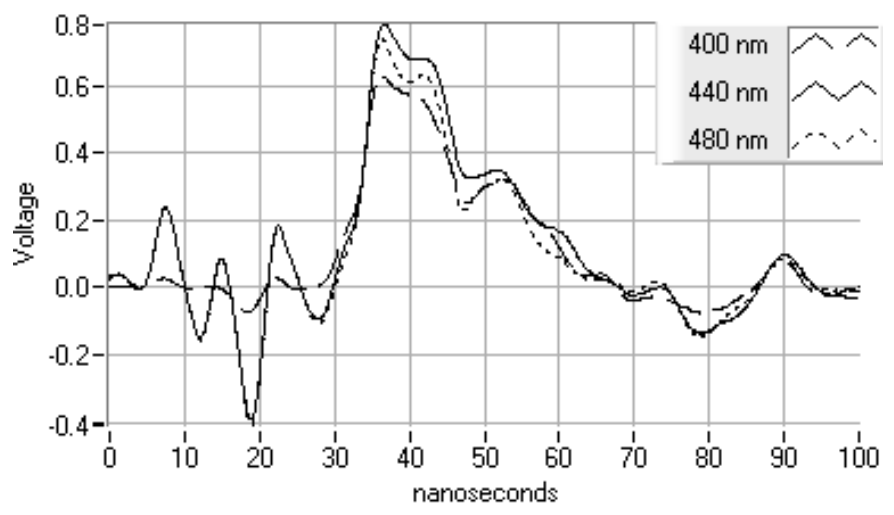

Figure 8. Time-resolved signals emitted by white color bond paper at three wavelengths. 


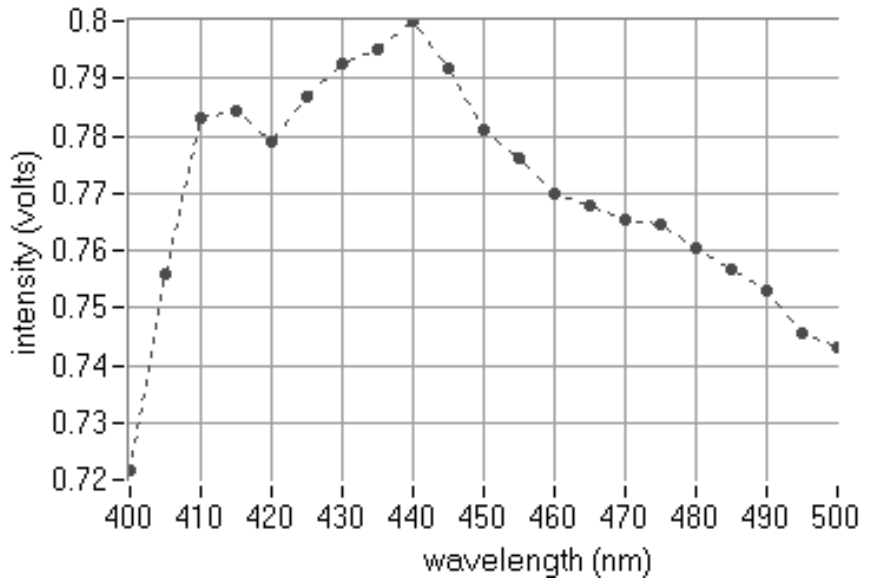

Figure 9. Florescence spectrum emitted by white color bond paper.

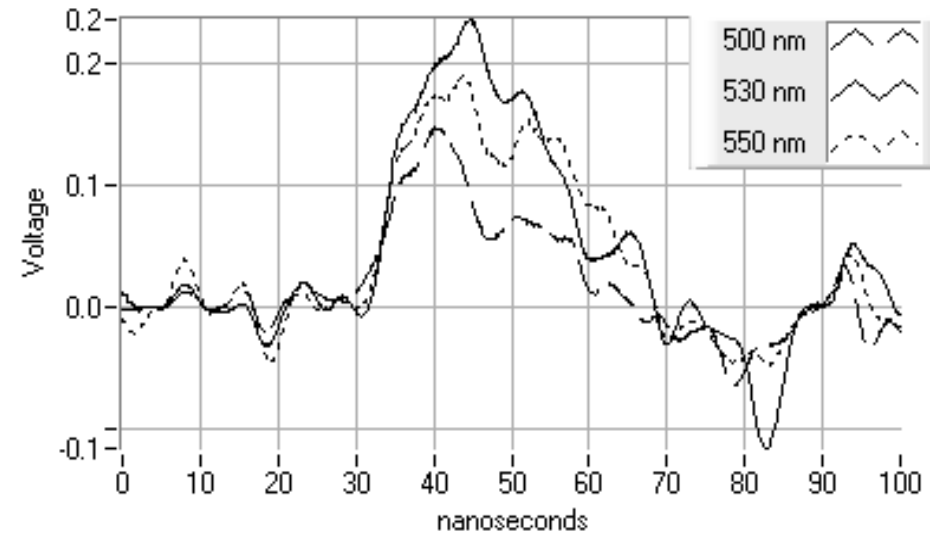

Figure 10. Time-resolved signals emitted by an antifreeze at three wavelengths.

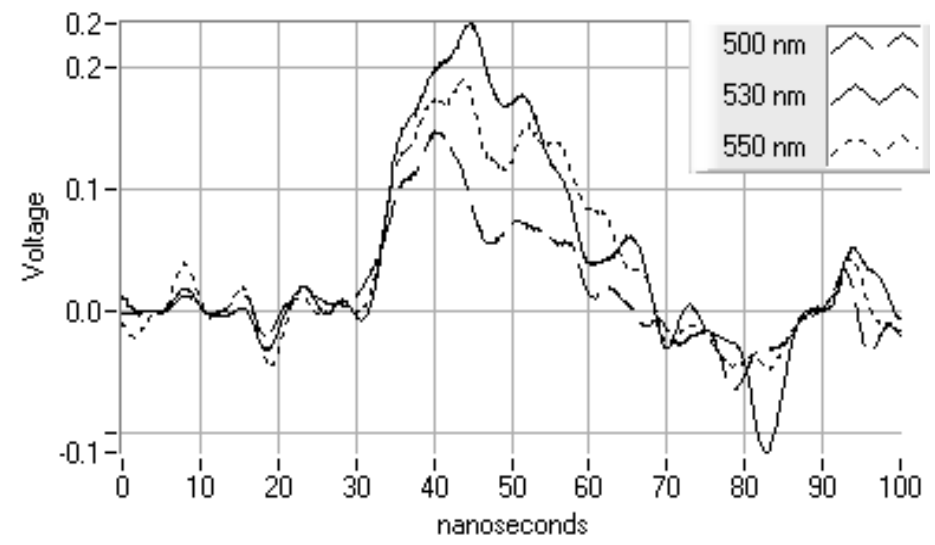

Figure 11. Florescence spectrum emitted by an antifreeze. 
Although the UV pulsed light source produces an unwanted oscillation, which limits the instrument capacity to measure fluorescence decays, it was possible to obtain the steady-state spectra of the substances from the intensities of time-resolved pulses. The fluorescence decays were acquired within $20 \mathrm{~ms}$ per wavelength with a SYNC pulse repetition rate of $40 \mathrm{KHz}$, but the time to transfer (via serial port) the data acquired from the DSP memory to the computer increases the acquisition rate near $4 \mathrm{~s} /$ wavelength.

The time resolution (150 ps) and the analog input bandwidth $(759 \mathrm{MHz})$ of the digitizer developed fulfill, at low cost, the requirements to be applied in nanosecond time-resolved fluorescence spectroscopy. For this application many authors have used commercial instrumentation to sample and to digitize the analog fluorescence signals, based on digital oscilloscopes, streak cameras, or other transient digitizers. Campos and Lytle [15] used a commercial waveform digitizer (Sequence, Model 3100), which has $1 \mathrm{GS} / \mathrm{s}$ sampling rate, 350 $\mathrm{MHz}$ bandwidth, a 10-bit static vertical resolution, and an effective data acquisition rate of 2.5 waveforms $/ \mathrm{s}$ ( $0.4 \mathrm{~s} /$ waveform). In their work they report fluorescence decay measurements with $1 \mathrm{~ns}$ resolution. All of these performance characteristics, except acquisition rate, are improved with our prototype. Recently, Muretta et al [16] reported a complete fluorescence decay curve recording for every excitation light pulse by means of a very fast but very expensive digitizer (Acqiris DC252 from Agilent Technologies). This modern instrument has 2 channels 10-bit amplitude resolution with programmable range, $2 \mathrm{GHz}$ bandwidth front end, 8 $\mathrm{GS} / \mathrm{s}$ real-time sampling rate, maximum time resolution of $0.125 \mathrm{~ns} /$ point, high recording rate of 500000 waveforms/s, and high acquisition rate of $0.1 \mathrm{~ms} /$ wavelength. The DC252 uses an interleaving of multiple ADCs to increase the effective sample rate by acquiring the same signal with multiple ADCs in parallel and out of phase.

Other authors have developed time-resolved spectrofluorometers to measure simultaneously fluorescence spectra and transient decays. Fang et al [7] digitized and recorded fluorescence decays by means of a digital oscilloscope (TDS5104 Tektronix) with $5 \mathrm{GS} / \mathrm{s}$ real-time sampling rate, 1 $\mathrm{GHz}$ front-end bandwidth, and 100,000 waveforms/s capture rate. They reported fluorescence decays acquired by the oscilloscope within $0.8 \mathrm{~s}$ per wavelength, including averaging of 16 consecutive pulses at laser repetition rate of 30 $\mathrm{Hz}$, digitization of the fluorescence decay pulse, data storage, and preliminary analysis. Pitts and Mycek [17] also used a digital oscilloscope (TDS$680 \mathrm{C}$ Tektronix) to capture fluorescence intensity as a function of time with similar characteristics as above. Although both oscilloscopes acquire data and perform some mathematical operations on waveforms at high rates, data have to be sent to a computer or other equipment for final processing and analysis. Thus the acquisition rate to register data on the computer is incremented by the transmission rate of the interface used (RS-232 or GPIB for both oscilloscopes).

Glanzmann et al [18] reported the use of a streak camera (C4792 Hamamatsu) coupled with a spectrograph as time-resolved spectrfluorometer. Florescence lifetimes from the sub ns up to the ms range can be measured. The images of the charge coupled device-chip in the streak camera are read out via a PCl-framegrabber board by a computer enabling data transfer in video rate $(30 \mathrm{~Hz})$. Terzic et al. [19] used state-of-the-art equipment from Hamamatsu. It is a complete streak system for fluorescence lifetime spectroscopy. The fluorescence decays are detected by a streakscope (C4334-01) with integrated video streak camera. The streakscope enables a wide range of fluorescence lifetime measurement from $\mathrm{ps}$ to $\mathrm{ms}$ with high accuracy and temporal resolution better than $15 \mathrm{ps}$, and with effective acquisition rate up 500000 waveforms/s.

Real-time methods for measuring such waveforms with subnanosecond time resolution, utilizing streak cameras or transient digitizers can be an order of magnitude more costly than equivalent-time methods using digital oscilloscopes or boxcar integrators. This equipment has been used as a relatively inexpensive solution for determining fluorescence lifetimes in the subnanosecond to nanosecond range. The prototype designed in this work can be an order of magnitude less costly than a digital oscilloscope, providing 150 ps temporal resolution and $750 \mathrm{MHz}$ front-end bandwidth (75 percent of $1 \mathrm{GHz}$ ). 


\section{Conclusions}

In this work we described the development of a high speed measurement system. All major components were selected such that they can be easily interfaced between them and with a computer. The prototype is reliable and useful for time-resolved analog pulses up to 2 ns rise time with a resolution of $150 \mathrm{ps}$ and an error less that 3 percent. The digitizer developed has an analog bandwidth front end of $750 \mathrm{MHz}$ which introduces an amplitude error of 5 percent for an input signal bandwidth of $175 \mathrm{MHz}$ or equivalent rise time of 2 ns. Three test pulses were measured with the digitizer and with a $300 \mathrm{MHz}$ bandwidth oscilloscope for comparison purposes. The measurements reported shows clearly that the digitizer developed resolves well-defined pulses within the expected values, meanwhile the oscilloscope distorts the input pulse and slows the rise time. The limited bandwidth of the oscilloscope cannot reproduce the highest frequency components of the test pulses. The equivalent-time sampling technique is functional and its algorithm can easily incorporate a simple averaging method for noise suppression. A control program was developed in the graphical language $\mathrm{G}$ of LabVIEW 7 to start a complete temporal sweep from 0 ns to 114.75 ns.

The developed prototype was applied to timeresolved nanosecond fluorescence pulses. Several substances were irradiated with UV light pulses at $365 \mathrm{~nm}$, and their luminous responses were detected with a homemade spectrometer. For this application the control program was improved to perform a wavelength sweep in an interval defined by the user. For each value of $\lambda$, the program commands the digitizer to execute a complete temporal sweep. Although the UV pulsed light source produces an unwanted oscillation, which limits the instrument capacity to measure fluorescence decays, it was possible to obtain the steady-state spectra of the substances from the intensities of the time-resolved pulses. From the measurements we concluded that the time resolution and bandwidth of the digitizer developed complies with the requirements to be applied in nanosecond time-resolved fluorescence spectroscopy.

\section{References}

[1] Lakowicz Joseph R., "Principles of Fluorescence Spectroscopy", Springer Science Business Media, New York, 3rd Edition, 2006.

[2] Gore Michael G., "Spectrophotometry and Spectrofluorimetry", Oxford University Press, 2nd Edition UK, 2000.

[3] C. Eichhorn, S. Fritzsche, S. Lôhle, A. Knapp, and M. Auweter-Kurtz, Time-resolved fluorescence spectroscopy of two-photon laser-excited 8p, 9p, $5 f$ and $6 f$ levels in neutral xenon, Phys. Rev. E. Stat Nonlin. Soft. Matter Phys. Vol. 80 (2Pt 2) Aug. 2009,

[4] S. Lehmann, G. Geipel, G. Grambole, and G. Bernhard, A novel time-resolved laser fluorescence spectroscopy system for research on complexation of uranium (IV), Spectrochimica Acta Part A: Molecular and Biomolecular Spectroscopy, Vol. 73, 9, September 2009, 902-908.

[5] N. Saitoh and S. Takeuchi, Fluorescence imaging of petroleum accelerants by time resolved spectroscopy with a pulsed Nd-Yag laser, Foresenic Science International, Vol. 163, 1-2, Nov. 2006, 38-50.

[6] Paras N. Prasad, "Introduction to Biophotonics", Jhon Wiley\&Sons, Inc., 2003.

[7] Q. Fang, T. Papaioannou, J. A. Jo, R. Vaita, and K Shastry, Time-domain laser-induced fluorescence spectroscopy apparatus for clinical diagnostics, Review of Scientific Instruments, Vol 75, 1, January 2004, 152162.

[8] P. V. Butte, A. N. Mamelak, M. Nuno, S. I. Bannykh, K. L. Black, and L. Marcu, Fluorescence lifetime spectroscopy for guided therapy of brain tumors, Neuroimage , Vol. 54, 2011, S125-S135.

[9] J. D. Meier, H. Xie, Y. Sun, Y. Sun, N. Hatami, B. Poirier, L. Marcu, and D. G. Farwell, Time resolved laser-induced fluorescence spectroscopy as a diagnostic instrument in head and neck carcinoma, Otolaryngology-Head and Neck surgery, Vol. 142, 6, June 2010, 838-844.

[10] Suzanne J. Lassiter, Wieslaw J. Stryjewski, Yun Wang, and Steven A. Soper, Fluorescence Lifetime Methods, 14 Spectroscopy 17(6) June 2002, www.spectroscopyonline.com. 
[11] E. Moreno-García, R. Rosas-Vélez, P. Reyes-López, J. de la Rosa-Vázquez, Development of a spectrometer controlled by a PC, Científica, Vol 11, No 3, Julio-Sept 2007, 149-154.

[12] R. Lawton, S. Riad, J. Andrews, Pulse \& Time- Domain Measurements,. Proc. IEEE, vol.74, Jan. 1986. $77-81$.

[13] N.S. Nahman, Picosecond-Domain Waveform Measurements, Proceedings of IEEE, Vol. 66, No.4, April 1978, 441-454.

[14]. Wilmshurst, T.H., "Signal Recovery from Noise in Electronic Instrumentation", 2nd Edition, Institute of Physics Publishing, Bristol, Great Britain, 1990.

[15] T. L. Campos and F. E. Lytle, Fluorometric Data Processing Strategies Using Nanosecond Waveform Digitizers, Applied Spectroscopy, Volume 46, Number 12, 1992, 1859-1865.

[16] J. M. Muretta, A. Kyrychenko, A. S. Ladokhin, D. J. Kast, G. D. Gillispie, and D. D. Thomas, A Highperformance time-resolved fluorescence by direct waveform recording, Rev. Sci. Instrum. 81, 2010, 103101-1- 103101-8.

[17] Jonathan D. Pitts and Mary-Ann Mycek, Design and development of a rapid acquisition laser-based fluorometer with simultaneous spectral and temporal resolution, Rev Sci. Instrum., Vol 72, No 7, July 2001, 3061-3072.

[18] T. Glanzmann, J-P Ballini, H. van den Bergh, and G. Wagnièresa, Time-resolved spectrofluorometer for clinical tissue characterization during endoscopy, Rev. Sci. Instrum., Vol. 70, No. 10, October 1999, 4067-4077.

[19] M. Terzić, B.P. Marinkocić, D, Servić, J, Jureta, and A.R.Milosavijević, Development of time-resolved laserinduced fluorescence spectroscopic technique for analysis of biomolecules, Facta Universitatis, Physics, Chemistry and Technology, Vol6, No. 1, 2008, 105-117 